\title{
A REDUCED BASIS METHOD FOR CONTROL PROBLEMS GOVERNED BY PDES *
}

\author{
K. ITO $^{\dagger}$ AND S.S. RAVINDRAN ${ }^{\dagger}$
}

\begin{abstract}
This article presents a reduced basis method for constructing a reduced order system for control problems governed by nonlinear partial differential equations. The major advantage of the reduced basis method over others based on finite element, finite difference or spectral method is that it may capture the essential property of solutions with very few basis elements. The feasibility of this method is demonstrated for boundary control problems modeled by the incompressible Navier-Stokes equations with the boundary temperature control and boundary electromagnetic control in channel flows.
\end{abstract}

Key words. reduced basis method, finite elements, optimal control, flow control.

AMS subject classifications. 93B40, 49M05, 76D05, 49K20, 65H10, 76W05, 80A20

\section{Contents}

1. Introduction.

2. Reduced Basis Spaces and Reduced-order Equation.

3. Error Analysis.

4. Optimal Control Problems.

5. The Reduced Basis Method for Flow Control.

5.1 Electrically Conducting Flow Equations and Variational Formulation. 5.2 Thermally Convective Flow Equations and Variational Formulation.

5.3 Mixed Finite Element Approximation.

5.4 Boundary Control Problems and Reduced-Order Control Problems.

6. Computational Results.

6.1 Boundary Temperature Control.

6.2 Electromagnetic Control.

1. Introduction. Real time simulations of control problems that involve partial differential equations as state equations are often formidable problems to solve. One such situation arises in control of fluid dynamical systems in which the state equations are the Navier-Stokes equations, the geometry is often complex and the time interval involved is often very large. If one were to solve such problems using standard finite element or finite difference method the resulting system is prohibitively large.

We in this article discuss a reduction type method which overcomes this difficulty. This method hereafter we call reduced basis method uses functions as basis functions which are closely related to and generated from the problem that is being solved. This is in

* This work was supported in part by the Air Force Office of Scientific Research under grants AFOSR F49620-95-1-0437 and AFOSR F49620-95-1-0447.

$\dagger$ Center for Research in Scientific Computation, Department of Mathematics, North Carolina State University, Raleigh, NC 27695-8205 (kitodeos.ncsu.edu and ravi@eos.ncsu.edu). 
contrast to the traditional numerical methods such as finite difference method which uses grid functions as basis functions or finite elements method which uses piecewise polynomials for this purpose.

There are several approaches available for the selection of basis functions. One such approach is Taylor approach in which one uses solutions at a reference point in the parameter space along with their derivatives as basis functions. Another approach which we call Lagrange approach uses solutions of the problem at various parameter values as basis functions. Finally the Hermite approach is a hybrid of Lagrange and Taylor approaches which uses solutions and their first derivatives of the problem at various parameter values as basis functions. The applications of reduced basis method to structural mechanics problems can be found in [1] and [6]-[7].

Our goal here is to demonstrate the applicability and feasibility of reduced basis method for control problems governed by Navier-Stokes type partial differential equations. We will consider vorticity minimization problems in backward-facing-step type channel geometry. Two fluid flow situations are considered: An electrically conducting fluid under applied magnetic field and a thermally convective fluid. In the first situation the control is effected by boundary electric potential and in the latter the control is boundary temperature.

2. Reduced Basis Spaces and Reduced-Order Equation. In order to illustrate the reduced basis method, we assume for ease in exposition that we are dealing with nonlinear dynamics about the equilibrium points. Consider the the parameterized stationary problem

$$
\mathcal{E}(y, \mu)=0 \quad \text { for } \mu \in \Lambda, y \in X,
$$

where $\mu$ represents some physical parameter, for example, Reynolds number or viscosity and $E: X \times \Lambda \rightarrow X^{*}$ is $C^{2}$. Equation (2.1) defines a solution function $\mu \in \Lambda \rightarrow y(\mu) \in X$. We construct the reduced basis elements by the interpolation of solution function $\mu \rightarrow y(\mu)$ as follows.

The Taylor Subspace. In this choice, one uses the Taylor expansion of function $y(\mu)$ at a reference value of $\mu$, say $\mu^{*}$, and the reduced basis subspace $X_{R}$ is defined as

$$
X_{R}=\operatorname{span}\left\{y_{j}\left|y_{j}=\frac{\partial^{j} y}{\partial \mu^{j}}\right|_{\mu=\mu^{*}}, j=0, \ldots, M\right\}
$$

The jth derivative $y_{j}$ can be calculated from the equations resulted by successive differentiation of (1.1), i.e.

$$
\mathcal{E}_{y}\left(y_{0}, \mu_{0}\right) y_{j}=\mathcal{F}_{j}\left(y_{0}, y_{1}, \ldots, y_{j-1}, \mu^{*}\right)
$$

For example, $y_{1}$ satisfies the equation

$$
\mathcal{E}_{y}\left(y_{0}, \mu^{*}\right) y_{1}=-\mathcal{E}_{\mu}\left(y_{0}, \mu^{*}\right) .
$$

We note here that each $y_{j}$ can be obtained from its predecessors by solving a linear system with the same linear operator $\mathcal{E}_{y}\left(y_{0}, \mu^{*}\right)$. However, one cannot continue to use the same 
basis elements generated at fixed parameter $\mu^{*}$ to compute solutions when the parameter of interest is significantly away from it. In such cases reduced basis elements have to be updated and the solution is sought in the new reduced basis space. Moreover, generating the right hand side of (2.3) could be quite complicated in certain problems. This choice has been extensively used in the literature, see for e.g [7] for structural analysis problems and [8] for high Reynolds number steady state fluid flow calculations.

The Lagrange Subspace. In this case, the basis elements are solutions of the nonlinear problem under study at various parameter values $\mu_{j}$. The reduced subspace is given by

$$
X_{R}=\operatorname{span}\left\{y^{j} \mid y^{j}=y\left(\mu_{j}\right), j=1, \ldots, M\right\} .
$$

The Lagrange basis was used to study structural problems in [1]. A possible advantage in this choice is that updating the basis elements can be done one basis element at a time instead of generating the whole space.

The Hermite Subspace. This is a hybrid of the Lagrange and Taylor approach. The basis elements are solutions and their first derivatives at various parameter values $\mu_{j}$. The reduced subspace is given by

$$
X_{R}=\operatorname{span}\left\{y^{j}=y\left(\mu_{j}\right) \text { and }\left.\frac{\partial y}{\partial \mu}\right|_{\mu=\mu_{j}}, j=1, \ldots, \widetilde{M}\right\} .
$$

Suppose we have a reduced basis space $X_{R}$ in $X$. Let $m=\operatorname{dim}\left(X_{R}\right)$ and $\left\{\phi_{i}\right\}$ is a basis of $X_{R}$. Then we can construct the reduced-order equation by the Galerkin approximation, i.e., for $y_{m}=\sum_{i=1}^{m} \alpha_{i} \phi_{i} \in X_{R}$

$$
\mathcal{E}_{m}\left(y_{m}, \mu\right)_{i}=\left\langle\mathcal{E}\left(y_{m}, \mu\right), \phi_{i}\right\rangle_{X * \times X}
$$

for $1 \leq i \leq m$.

For the evolution equation

$$
\frac{d}{d t} y(t)+E(t, y(t))=0
$$

we, for example, generate reduced basis elements $\left\{\phi_{i}\right\}_{i=1}^{m}$ in $X$ by the solutions at $m$ different time instants to (2.7). Given the reduced basis space $X_{R}$, we define the reduced-order equation for $y_{m}(t)=\sum_{i=1}^{m} \alpha_{i}(t) \phi_{i}$,

$$
\left\langle\frac{d}{d t} y_{m}(t)+E\left(t, y_{m}(t)\right), \phi_{i}\right\rangle_{X * \times X}=0
$$

for all $1 \leq i \leq m$. In [4] this method has been carried out and its feasibility has been demonstrated for channel flow simulations in which reduced order solution $\mathbf{u}^{m}$ is formed by setting

$$
\mathbf{u}^{m}(t)=\sum_{i=1}^{m} \alpha_{i}(t) \phi_{i},
$$


where $\boldsymbol{\phi}_{i}=\mathbf{u}_{i+1}-\mathbf{u}_{i}, i=1,2, \ldots, m-1$ and $\boldsymbol{\phi}_{m}=\mathbf{u}_{m}$. We further take $\alpha_{m}=1$ so that the boundary conditions are satisfied. The solution $\mathbf{u}^{m}$ is computed from

$$
\left(\frac{\partial}{\partial t} \mathbf{u}^{m}, \mathbf{v}^{m}\right)+\frac{1}{R e} a\left(\mathbf{u}^{m}, \mathbf{v}^{m}\right)+c\left(\mathbf{u}^{m}, \mathbf{u}^{m}, \mathbf{v}^{m}\right)=\left(\mathbf{f}, \mathbf{v}^{m}\right) \text { for all } \mathbf{v}^{m} \in \mathbf{V}_{0}^{m},
$$

where $\mathbf{V}_{0}^{m}=\operatorname{span}\left\{\boldsymbol{\phi}_{i}: i=1, \ldots, m-1\right\}$ is the span of the test functions.

The basis elements were generated by computing the flow from the full model at eleven time instances between 1 and 11. The time step used in the reduced order model was .001 .

The dimension of the reduced basis space is very much problem-dependent. The reduced basis elements constructed by the above mentioned approach can be nearly linearly dependent. So we may further reduce the dimension by the conditioning of the mass matrix $Q$ :

$$
Q_{i, j}=\left(\phi_{i}, \phi_{j}\right)_{X}
$$

Our computational experiments and the computations reported for structural problems in the references mentioned earlier seem to indicate that an accurate approximation can be obtained for large range of parameter values using 5 to 10 basis elements. Therefore, although the resulting reduced order model is dense, they are small compared to the sparse but large system that result from the standard basis functions.

According to our comparison study carried out in [4] for driven cavity incompressible Navier-Stokes calculations, the performance of Hermite approach is better than that of Lagrange. The basis elements for the Lagrange approach were selected at Reynolds numbers $100,300,500$ and 700 , and that for the Hermite was selected at 300 and 700 . The comparison was carried out by computing the driven cavity flow at Reynolds number 1200 . The $L_{2}$-norm difference between the full mixed-finite element solution $u_{f}$ and the reduced basis solution using these two approaches are as follows: $\left\|\mathbf{u}_{l}-\mathbf{u}_{f}\right\|_{2}=0.0889$ and $\left\|\mathbf{u}_{h}-\mathbf{u}_{f}\right\|_{2}=0.0766$, where $\mathbf{u}_{l}$ is the solution obtained using Lagrange approach and $\mathbf{u}_{h}$ is that obtained using Hermite approach.

3. Error Analysis. In order to justify the reduced basis solution $y_{m}$ we need to have a post verification criterion. In general we formulate it as an error analysis as follows. Let $\mathrm{X}$ and $\mathrm{Y}$ be two Banach spaces and $\Lambda$ be a compact set. Given a $C^{2}$ mapping

$$
\mathcal{E}:(y, \mu) \in X \times \Lambda \rightarrow \mathcal{E}(y, \mu) \in Y,
$$

and we consider the equation

$$
\mathcal{E}(y, \mu)=0 .
$$

The family $\{(y(\mu), \mu): \mu \in \Lambda\}$ is said to be a branch of nonsingular solutions of equation (3.1), i.e.,

$$
\begin{gathered}
\mu \rightarrow y(\mu) \text { is a continuous function from } \Lambda \text { into } X \text { and } D_{y} \mathcal{E}(y, \mu) \text { is } \\
\text { an isomorphism from } X \text { onto } Y \text { for all } \mu \in \Lambda \text {. }
\end{gathered}
$$


Let us consider the reduced order problem

$$
\mathcal{E}_{m}\left(y_{m}, \mu\right)=0
$$

defined on the reduced basis space $X_{n}$. We assume that $\mathcal{E}_{m}: X_{m} \times \Lambda \rightarrow Y_{m}$ is $C^{2}$. For the ease of our discussions we assume that $X_{m} \subset X$ and $Y_{m} \subset Y$. The norms on $X_{m}$ and $Y_{m}$ are induced from $X$ and $Y$ norms, respectively. The problem is to find the solution $y_{m} \in X_{m}$ such that (3.2) is satisfied for a given $\mu \in \Lambda$.

We assume that $D_{y} \mathcal{E}_{m}\left(\tilde{y}_{m}, \mu\right)$ is an isomorphism from $X_{m}$ onto $Y_{m}$ where $\tilde{y}_{m}$ is a given element in $X_{m}$. We introduce the following notations;

$$
\begin{aligned}
& \epsilon_{m}(\mu)=\left\|\mathcal{E}_{m}\left(\tilde{y}_{m}, \mu\right)\right\|_{Y_{m}}, \\
& \gamma_{m}(\mu)=\left\|D_{y} \mathcal{E}_{m}\left(\tilde{y}_{m}, \mu\right)^{-1}\right\|_{\mathcal{L}\left(Y_{m}, X_{m}\right)} \\
& S_{m}(y ; \alpha)=\left\{v \in X_{m}:\|u-v\|_{X_{m}} \leq \alpha\right\} \\
& L_{m}(\mu ; \alpha)=\sup _{v \in S\left(\tilde{y}_{m}, \alpha\right)}\left\|D_{y} \mathcal{E}_{m}\left(\tilde{y}_{m}, \mu\right)-D_{y} \mathcal{E}_{m}(v, \mu)\right\|_{\mathcal{L}\left(X_{m}, Y_{m}\right)}
\end{aligned}
$$

We next state a theorem regarding the error estimate which is derived from Theorem IV.3.1 in [3] for the approximation of branches of nonsingular solutions.

Theorem 3.1. Suppose $D_{y} \mathcal{E}_{m}\left(\tilde{y}_{m}, \mu\right)$ is an isomorphism of $X_{m}$ onto $Y_{m}$ and

$$
2 \gamma_{m}(\mu) L_{m}\left(\mu, 2 \gamma_{m}(\mu) \epsilon_{m}(\mu)\right)<1 .
$$

Then the problem (3.2) has a unique solution $\left(y_{m}(\mu), \mu\right)$ such that:

$$
y_{m}(\mu) \in S\left(\tilde{y}_{m} ; 2 \gamma_{m}(\mu) \epsilon_{m}(\mu)\right) .
$$

In addition, $y_{m}(\mu)$ is the only solution of (3.2) in the ball $S_{m}\left(\tilde{y}_{m} ; \alpha\right)$ for all $\alpha \geq 2 \gamma_{m}(\mu) \epsilon_{m}(\mu)$ that satisfy $\gamma_{m}(\mu) L_{m}(\mu ; \alpha)<1$ and we have the estimate:

$$
\left\|y_{m}(\mu)-v_{m}\right\|_{X} \leq\left[\gamma_{m}(\mu) /\left(1-\gamma_{m}(\mu) L_{m}(\mu ; \alpha)\right]\left\|\mathcal{E}_{m}\left(v_{m}, \mu\right)\right\|_{Y} \quad \text { for all } v_{m} \in S_{m}\left(\tilde{y}_{m}, \alpha\right) .\right.
$$

Moreover, we have the following corollary.

Corollary 3.2. Suppose there exists an element $\tilde{y}_{m} \in X_{m}$ such that $D_{y} \mathcal{E}\left(\tilde{y}_{m}, \mu\right)$ is an isomorphism of $X$ onto $Y$ and

$$
2 \gamma(\mu) L(\mu, 2 \gamma(\mu) \epsilon(\mu))<1
$$

where

$$
\begin{aligned}
& \epsilon(\mu)=\left\|\mathcal{E}\left(\tilde{y}_{m}, \mu\right)\right\|_{Y}, \\
& \gamma(\mu)=\left\|D_{y} \mathcal{E}\left(\tilde{y}_{m}, \mu\right)^{-1}\right\|_{\mathcal{L}(Y, X)}, \\
& S(y ; \alpha)=\left\{v \in X:\|u-v\|_{X} \leq \alpha\right\}, \\
& L(\mu ; \alpha)=\sup _{v \in S\left(\tilde{y}_{m}, \alpha\right)}\left\|D_{y} \mathcal{E}\left(\tilde{y}_{m}, \mu\right)-D_{y} \mathcal{E}(v, \mu)\right\|_{\mathcal{L}(X, Y)} .
\end{aligned}
$$


Then the problem (3.1) has a solution $(y(\mu), \mu)$ such that:

$$
y(\mu) \in S\left(\tilde{y}_{m} ; 2 \gamma(\mu) \epsilon(\mu)\right) .
$$

In addition, $y(\mu)$ is the only solution of (3.1) in the ball $S\left(\tilde{y}_{m} ; \alpha\right)$ for all $\alpha \geq 2 \gamma(\mu) \epsilon(\mu)$ that satisfy $\gamma(\mu) L(\mu ; \alpha)<1$ and we have the estimate:

$$
\|y(\mu)-v\|_{X} \leq\left[\gamma(\mu) /(1-\gamma(\mu) L(\mu ; \alpha)] \mid \mathcal{E}(v, \mu) \|_{Y}\right.
$$

for all $v \in S\left(\tilde{y}_{m}, \alpha\right)$.

We can apply Theorem 3.1 and Corollary 3.2 to obtain the following error estimate.

Theorem 3.3. (i) Suppose $y_{m}(\mu) \in X_{m}$ is a solution to (3.2) and assume $\tilde{y}_{m}=y_{m}(\mu)$ satisfies the condition in Corollary 3.2. Then we have a solution $y(\mu) \in S\left(\tilde{y}_{m} ; 2 \gamma(\mu) \epsilon(\mu)\right)$ to (3.1) and the estimate

$$
\| y(\mu)-\left.y_{m}(\mu)\right|_{X} \leq\left[\gamma(\mu) /(1-\gamma(\mu) L(\mu ; \alpha)] \mid \mathcal{E}\left(y_{m}(\mu), \mu\right) \|_{Y}\right.
$$

with $\alpha=\gamma(\mu) \epsilon(\mu)$.

(ii) Suppose there exits an element $\tilde{y}_{m} \in X_{m}$ such that the conditions in Theorem 3.1 and Corollary 3.2 are satisfied. Furthermore, we assume that $\alpha_{m}=2 \gamma_{m}(\mu) \epsilon_{m}(\mu)$ satisfies $\gamma(\mu) L\left(\mu ; \alpha_{m}\right)<1$. Then we have (3.4) with $\alpha=\alpha_{n}$.

4. Optimal Control Problems. In this section we discuss the optimal control problem and the application of reduced basis method. Consider the minimization problem

$$
\min J(y, u) \text { subject to } E(y, u)=0
$$

over $u \in U_{a d} \subset U$. Here $\mathrm{X}$ and $\mathrm{U}$ are Hilbert spaces and $E: X \times U \rightarrow X^{*}$ is $C^{2}$. We assume that $U=R^{m}$ and $u_{a d}$ is closed and convex. The Lagrange reduced space can be defined by

$$
X_{R}=\operatorname{span}\left\{y^{j} \in X \mid E\left(y^{j}, u^{j}\right)=0, j=1, \ldots, M\right\},
$$

where $u^{j}$ is a sampled point in $U_{a d}$. In order to obtain a lower-order reduced basis space, if $m$ is large then we may consider the following pre-selecting step:

(1) let $u^{\alpha}, \alpha \in A$ be the points in $U_{a d}$ defined by

$$
u^{\alpha}=\bar{u}+\sum \delta_{i} \alpha_{i} e_{i}
$$

where $\alpha$ is the integer-valued vector, and $\delta_{i}$ and $e_{i}$ is the step size and unit vector in the $i$-th direction,

(2) we determine $y^{\alpha} \in X$ by solving $E\left(y, u^{\alpha}\right)$ for each $\alpha \in A$.

(3) we find an index $\alpha_{0}$ in $A$ such that $J\left(y^{\alpha}, u^{\alpha}\right)$ is smallest, and then

(4) we select the sampling set $u_{j}$ by

$$
u^{1}=u^{\alpha_{0}}, \quad u^{2 i}=u^{\alpha_{0}}+\delta_{i} e_{i}, \quad \text { and } \quad u^{2 i+1}=u_{\alpha_{0}}-\delta_{i} \epsilon_{i} .
$$


The Hermite reduced space can be defined by

$$
X_{R}=\operatorname{span}\left\{y^{j} \in X \times U \mid E\left(y^{j}, u^{j}\right)=0 \text { and } \frac{\partial y}{\partial u_{i}}\left(u^{j}\right), 1 \leq i \leq m, j=1, \ldots, \widetilde{M}\right\} .
$$

Here, $\xi_{i}^{j}=\frac{\partial y}{\partial u_{i}}\left(u_{j}\right)$ can be calculated by solving the sensitivity equation

$$
\mathcal{E}_{y}\left(y^{j}, u^{j}\right) \xi_{i}^{j}=-\mathcal{E}_{u_{i}}\left(y_{0}, u^{j}\right) .
$$

Here we can use the pre-selecting step to select $u^{j}$ as for the Lagrange case.

Suppose we have the reduced basis space $X_{R}$. Then we use the Galerkin method to project the equation onto $X_{R}$, i.e., $y^{m}=\sum_{i=1}^{m} \alpha_{i} \phi_{i} \in X_{R}$ satisfies

$$
\mathcal{E}_{m}\left(y^{m}, u\right)_{i}=\left\langle\mathcal{E}(y, u), \phi_{i}\right\rangle_{X^{*} \times X} .
$$

Then we consider the reduced-order control problem

$$
\min J\left(y^{m}, u\right) \text { subject to } \mathcal{E}_{m}\left(y^{m}, u\right)=0 \text { and } u \in U_{a d} \text {. }
$$

It is a finite dimensional constrained minimization problem and can be readily solved by the constrained optimization methods. A necessary optimality condition is given by

$$
\begin{aligned}
& \left(D_{y} \mathcal{E}_{m}\left(y_{m}, u_{m}\right)\right)^{t} \lambda_{m}+D_{y} J\left(y_{m}, u_{m}\right)=0 \\
& \left(D_{u} \mathcal{E}_{m}\left(y_{m}, u_{m}\right)\left(u-u_{m}\right), \lambda_{m}\right)+D_{u} J\left(y_{m}, u_{m}\right)\left(u-u_{m}\right) \geq 0
\end{aligned}
$$

for all $u \in U_{a d}$, assuming $D_{y} \mathcal{E}_{m}$ at the optimal pair $\left(y_{m}, u_{m}\right)$ to (4.3) is an isomorphism. Similarly, we have the necessary optimality condition for (4.1): there exists a Lagrange multiplier $\lambda \in X$ such that

$$
\begin{aligned}
& \left(D_{y} \mathcal{E}\left(y^{*}, u^{*}\right)\right)^{*} \lambda+D_{y} J\left(y^{*}, u^{*}\right)=0 \\
& \left\langle D_{u} \mathcal{E}\left(y^{*}, u^{*}\right)\left(u-u^{*}\right), \lambda\right\rangle_{X^{*} \times X}+D_{u} J\left(y^{*}, u^{*}\right)\left(u-u^{*}\right) \geq 0
\end{aligned}
$$

for all $u \in U_{a d}$, assuming $D_{y} \mathcal{E}_{m} \mathcal{L}\left(y^{*}, u^{*}\right)$ at the optimal pair $\left(y^{*}, u^{*}\right)$ to $(4.1)$ is an isomorphism. Suppose $u_{m}$ and $u^{*}$ is interior points of $U_{a d}$. Then we can apply Theorem 3.3 to equation for $(y, \lambda, u) \in X \times X \times U$

$$
\begin{aligned}
& E(y, u)=0 \\
& \left(D_{y} \mathcal{E}(y, u)\right)^{*} \lambda+D_{y} J(y, u)=0 \\
& \left(D_{u} \mathcal{E}(y, u)\right)^{*} \lambda+D_{u} J(y, u)=0
\end{aligned}
$$

In general we have

$$
J\left(y_{m}, u_{m}\right) \leq J\left(y^{m}(u), u\right)=J\left(y^{m}(u), u\right)-J(y(u), u)+J(y(u), u)
$$

for $u \in U_{a d}$ and thus setting $u=u^{*}$, we obtain

$$
J\left(y\left(u_{m}\right), u_{m}\right)-J^{*} \leq J\left(y\left(u_{m}\right), u_{m}\right)-J\left(y_{m}, u_{m}\right)+J\left(y^{m}\left(u^{*}\right), u^{*}\right)-J\left(y\left(u^{*}\right), u^{*}\right) .
$$

Hence, if $U_{a d}$ is compact then

$$
J\left(y\left(u_{m}\right), u_{m}\right)-J^{*} \leq 2 M \max _{u \in u_{a d}}\left\|y^{m}(u)-y(u)\right\|_{X}
$$

for some constant $M$. 
5. The Reduced Basis Method for Flow Control. In this section we discuss vorticity minimization in fluid flows using boundary temperature control and electromagnetic control. We first present the weak variational formulation of the optimal control problems and then discuss their approximations in finite element and reduced basis setting.

5.1. Electrically Conducting Flow Equations and Variational Formulation. In this section we describe the governing equations for an electrically conducting flow and their variational formulation. Suppose there is a length scale $\ell$, a velocity scale $\mathbf{U}$ and a magnetic-field scale $B_{0}$ in the flow, then one can define nondimensional magnetic Reynolds number $R_{m}=\mu_{0} \sigma U \ell$, where $\mu_{0}$ is the magnetic permiability, Alfven number $A l=B_{0}^{2} / \mu_{0} \rho U^{2}$ and Reynolds number $R e=\rho_{0} U \ell / \mu$. Next, if we nondimensionalize according to $\mathbf{x} \leftarrow \mathbf{x} / \ell, \mathbf{u} \leftarrow \mathbf{u} / \mathbf{U}, \mathbf{j} \leftarrow B_{0} / \ell, \mathbf{E} \leftarrow U B_{0}$ and $p \leftarrow(p-\mathbf{g} \cdot \mathbf{x}) /\left(\rho_{0} \mathbf{U}^{2}\right)$, we obtain the dimensionless equations $\Omega$;

$$
\begin{aligned}
& \mathbf{u} \cdot \nabla \mathbf{u}=-\nabla p+A l(\mathbf{j} \times \mathbf{B})+\frac{1}{R e} \Delta \mathbf{u} \text { and } \nabla \cdot \mathbf{u}=0 \\
& \mathbf{j}=R_{m}[-\nabla \phi+(\mathbf{u} \times \mathbf{B})], \text { and } \nabla \cdot \mathbf{j}=0 \\
& \nabla \times \mathbf{B}=\mathbf{j} \text { and } \nabla \cdot \mathbf{B}=0 .
\end{aligned}
$$

Here, $\mathbf{u}$ denotes the velocity field, $p$ the pressure field, $\mathbf{j}$ the electric current density, $\mathbf{B}$ the magnetic field and $\phi$ the electric potential. We denote by $\Omega$ the flow domain which is a bounded in $R^{2}$ with boundary $\Gamma$.

Although the problems and methods studied here are applicable to the optimal control for general models of magneto-hydrodynamic flows, for the simplicity of explaining the ideas we will deal with a special case in which the externally applied magnetic field is undisturbed by the flow; i.e., we assume that $\mathbf{B}$ is given. Such an assumption can be met in a variety of physical applications, e.g., in the modeling of electromagnetic pumps and the flow of liquid lithium for fusion reactor cooling blankets (see for e.g. [10]). Under this assumption, the term $\mathbf{j} \times \mathbf{B}$ in the Navier-Stokes equations can be written as

$$
A l(\mathbf{j} \times \mathbf{B})=N(-\nabla \phi+\mathbf{u} \times \mathbf{B}) \times \mathbf{B}
$$

where $N=A l \cdot R_{m}$ and if we eliminate $\mathbf{j}$ by applying charge conservation condition $\nabla \cdot \mathbf{j}=0$ to $\mathbf{j}=R_{m}[-\nabla \phi+(\mathbf{u} \times \mathbf{B})]$, we arrive at the following simplified system modeling the flow:

$$
-\frac{1}{R e} \Delta \mathbf{u}+\mathbf{u} \cdot \nabla \mathbf{u}+\nabla p+N(\nabla \phi-\mathbf{u} \times \mathbf{B}) \times \mathbf{B}=0,
$$

$$
\begin{aligned}
& \nabla \cdot \mathbf{u}=0 \\
& -\Delta \phi+\nabla \cdot(\mathbf{u} \times \mathbf{B})=0
\end{aligned}
$$

where $N$ is the interaction parameter. The system (5.1) is supplemented with boundary conditions

$$
\mathbf{u}=\mathbf{u}_{0} \text { on } \Gamma, \quad \phi=g \text { on } \Gamma_{0} \text { and } \mathbf{n} \cdot \mathbf{j}=0 \text { on } \Gamma_{1}
$$


where $\Gamma$ is the disjoint union $\Gamma=\Gamma_{0} \cup \Gamma_{1}$ and $g$ denotes the control function, namely, electric potential on $\Gamma_{0}$. Such a control can be effected by attaching electric sources with adjustable resistors to the electrode along the boundary. We assume that the flow is two-dimensional and the applied magnetic field $\mathbf{B}$ is perpendicular to the flow plane, i.e., $\mathbf{B}=\left(0,0, B_{0}\right)^{t}$, and that the cross product $\mathbf{u} \times \mathbf{B}$ is understood as $\left(u_{1}, u_{2}, 0\right)^{t} \times\left(0,0, B_{0}\right)^{t}$. Let $\overline{\mathbf{u}} \in\left(H^{1}(\Omega)\right)^{2}$ and $\bar{\phi} \in H^{1}(\Omega)$ be such that

$$
\overline{\mathbf{u}}=\mathbf{u}_{0} \text { on } \Gamma \text { and } \bar{\phi}=g \text { on } \Gamma_{0}
$$

and $V_{1}=\left\{\phi \in H^{1}(\Omega): \phi=0\right.$ on $\left.\Gamma_{0}\right\}$. Then, variational formulation of (5.1)-(5.2) is given as follows: seek $\mathbf{u} \in\left(H_{0}^{1}(\Omega)\right)^{2}+\overline{\mathbf{u}}, p \in L^{2}(\Omega)$ and $\phi \in H_{0}^{1}(\Omega)+\bar{\phi}$ such that

$$
\begin{aligned}
& \frac{1}{R e}(\nabla \mathbf{u}, \nabla \mathbf{v})+b_{0}(\mathbf{u}, \mathbf{u}, \mathbf{v}) \\
& \quad+N(\nabla \phi-\mathbf{u} \times \mathbf{B}, \mathbf{v} \times \mathbf{B})-(p, \nabla \cdot \mathbf{v})=0, \quad \forall \mathbf{v} \in\left(H_{0}^{1}(\Omega)\right)^{2} \\
& (\nabla \cdot \mathbf{u}, q)=0, \quad \forall q \in L^{2}(\Omega) \\
& (\nabla \phi-\mathbf{u} \times \mathbf{B}, \nabla \psi)=0, \quad \forall \psi \in V_{1} .
\end{aligned}
$$

Here, the trilinear form $b_{0}$ on $\left(H^{1}(\Omega)^{2}\right)^{3}$ is defined by

$$
b_{0}(\mathbf{u}, \mathbf{v}, \mathbf{w})=\langle\mathbf{u} \cdot \nabla \mathbf{v}, \mathbf{w}\rangle
$$

for $\mathbf{u}, \mathbf{v}, \mathbf{w} \in\left(H^{1}(\Omega)\right)^{2}$.

5.2. Thermally Convective Flow Equations and Variational Formulation. In this section we describe the governing equations for a thermally conducting flow and their variational formulation.

If we assume there is a length scale $\ell$, a velocity scale $\mathbf{U}$ and a temperature scale $T_{1}-T_{0}$ in the flow, then one can define nondimensional Prandtl number $\operatorname{Pr}=\mu c_{p} / \kappa$, Grashof number $G r=\beta \ell^{3} \rho_{0}^{2}|\mathbf{g}|\left(T_{1}-T_{0}\right) / \mu^{2}$ and Reynolds number $R e=\rho_{0} U \ell / \mu$. Next, if we nondimensionalize according to $\mathbf{x} \leftarrow \mathbf{x} / \ell, \mathbf{u} \leftarrow \mathbf{u} / \mathbf{U}, T \leftarrow\left(T-T_{0}\right) /\left(T_{1}-T_{0}\right)$, and $p \leftarrow$ $(p-\mathbf{g} \cdot \mathbf{x}) /\left(\rho_{0} \mathbf{U}^{2}\right)$, we obtain the following nondimensional form of Boussinesq equations.

$$
\begin{aligned}
& -\frac{1}{R e} \Delta \mathbf{u}+(\mathbf{u} \cdot \nabla) \mathbf{u}+\nabla p+\frac{G r}{R e^{2}} T \mathbf{g}=\mathbf{0} \text { in } \Omega, \\
& \nabla \cdot \mathbf{u}=0 \quad \text { in } \Omega, \\
& -\frac{1}{R e P r} \Delta T+\mathbf{u} \cdot \nabla T=0 \quad \text { in } \Omega,
\end{aligned}
$$

where $\mathrm{g}$ is a unit vector in the direction of gravitational acceleration.

We consider the boundary condition as follows.

$$
\mathbf{u}=\mathbf{u}_{0} \text { on } \Gamma, \quad T=g \text { on } \Gamma_{0} \text { and } \frac{\partial T}{\partial \mathbf{n}}=0 \text { on } \Gamma_{1}
$$


where $g$ represents the boundary temperature control function. Let $\bar{T} \in H^{1}(\Omega)$ be such that $\bar{T}=g$ on $\Gamma_{0}$. Then, variational formulation of $(5.5)-(5.6)$ is given by

$$
\begin{aligned}
& \frac{1}{R e}(\nabla \mathbf{u}, \nabla \mathbf{v})+b_{0}(\mathbf{u}, \mathbf{u}, \mathbf{v})+(\beta T \mathbf{g}, \mathbf{v})-(p, \nabla \cdot \mathbf{v})=0, \quad \forall \mathbf{v} \in\left(H_{0}^{1}(\Omega)\right)^{2} \\
& (\nabla \cdot \mathbf{u}, q)=0, \quad \forall q \in L^{2}(\Omega) \\
& b_{1}(\mathbf{u}, T, \psi)+\kappa(\nabla T, \nabla \psi), \quad \forall \psi \in V_{1}
\end{aligned}
$$

for $\mathbf{u} \in\left(H_{0}^{1}(\Omega)\right)^{2}+\overline{\mathbf{u}}, p \in L^{2}(\Omega)$ and $T \in H_{0}^{1}(\Omega)+\bar{T}$, where $\beta=\frac{G r}{R e^{2}}, \kappa=\frac{1}{R e P r}$ and the trilinear form $b_{1}$ is given by

$$
b_{1}(\mathbf{u}, T, \psi)=\langle\mathbf{u} \cdot \nabla T, \psi\rangle .
$$

for $T, \psi \in H^{1}(\Omega)$ and $\mathbf{u} \in\left(H^{1}(\Omega)\right)^{2}$.

We can establish the existence of solutions to (5.3) and (5.7) (e.g, see [5]), using the above properties of the triliner forms and the Hopf's lemma.

5.3. Mixed Finite Element Approximation. In order to construct the reduced basis element we use the mixed finite element method [3] to approximate solution to (5.1)(5.2) and (5.5)-(5.6).

Let us define, using standard finite element notations,

$$
\begin{aligned}
& X^{h}=\left\{v \in C^{0}(\bar{\Omega}):\left.v\right|_{K} \in P_{2}(K), \text { on each element } K\right\}, \\
& \mathbf{X}^{h}=\left\{\mathbf{v}=\left(v_{1}, v_{2}\right)^{T} \in \mathbf{C}^{0}(\bar{\Omega}): v_{i} \in X^{h}, i=1,2\right\}
\end{aligned}
$$

and

$$
S^{h}=\left\{q \in C^{0}(\bar{\Omega}):\left.q\right|_{K} \in P_{1}(K) \text {, on each element } K\right\} .
$$

Also we define $\mathbf{X}_{0}^{h}=\left\{v \in \mathbf{X}^{h}: v=0\right.$ on $\left.\Gamma\right\}$ and $X_{1}^{h}=X^{h} \cap V_{1}$. That is, we choose continuous piecewise quadratic polynomials for both components of the velocity $\mathbf{u}^{h}$ and electric potential $\phi^{h}$ for (5.1) and temperature $T^{h}$ for (5.5), continuous piecewise linear polynomials for the pressure $p^{h}$. On each triangle, the degrees of freedom for quadratic elements are the function values at the vertices and midpoints of each edge; the degrees of freedom for linear elements are the function values at the vertices. Here, the spaces are defined over the same triangulation of the domain $\Omega=\bigcup K$. This selection is known as Taylor-Hood element pair and has been shown to satisfy the LBB or inf-sup condition.

The finite element equation of (5.3) for $\mathbf{u}^{h} \in \mathbf{X}^{h}, p^{h} \in S^{h}$ and $\phi^{h} \in X^{h}$ is given by

$$
\begin{aligned}
& \frac{1}{R e}\left(\nabla \mathbf{u}^{h}, \nabla \mathbf{v}^{h}\right)+b_{0}\left(\mathbf{u}^{h}, \mathbf{u}^{h}, \mathbf{v}^{h}\right) \\
& \quad+N\left(\nabla \phi^{h}-\mathbf{u}^{h} \times \mathbf{B}, \mathbf{v}^{h} \times \mathbf{B}\right)-\left(p^{h}, \nabla \cdot \mathbf{v}^{h}\right)=0, \quad \forall \mathbf{v}^{h} \in \mathbf{X}_{0}^{h} \\
& \left(\nabla \cdot \mathbf{u}^{h}, q^{h}\right)=0, \quad \forall q^{h} \in S^{h} \\
& \left(\nabla \phi^{h}-\mathbf{u}^{h} \times \mathbf{B}, \nabla \psi^{h}\right)=0, \quad \forall \psi^{h} \in X_{1}^{h} .
\end{aligned}
$$


where $\left.\mathbf{u}^{h}\right|_{\Gamma}=\mathbf{u}_{0}^{h}$ and $\left.\phi^{h}\right|_{\Gamma_{0}}=g^{h}$ and $\mathbf{u}_{0}^{h}, g^{h}$ are the projection of $\mathbf{u}_{0}, g$ onto the finite element spaces, respectively. Similarly, for the Boussinesq equation (5.7) we have

$$
\begin{aligned}
& \frac{1}{R e}\left(\nabla \mathbf{u}^{h}, \nabla \mathbf{v}^{h}\right)+b_{0}\left(\mathbf{u}^{h}, \mathbf{u}^{h}, \mathbf{v}^{h}\right)+\left(\beta T^{h} \mathbf{g}, \mathbf{v}^{h}\right)-\left(p^{h}, \nabla \cdot \mathbf{v}^{h}\right)=0, \quad \forall \mathbf{v}^{h} \in \mathbf{X}_{0}^{h} \\
& \left(\nabla \cdot \mathbf{u}^{h}, q^{h}\right)=0, \quad \forall q^{h} \in S^{h} \\
& b_{1}\left(\mathbf{u}^{h}, T^{h}, \psi^{h}\right)+\kappa\left(\nabla T^{h}, \nabla \psi^{h}\right), \quad \forall \psi^{h} \in X_{1}^{h} .
\end{aligned}
$$

where $\mathbf{u}^{h} \in \mathbf{X}^{h}, T^{h} \in X^{h}$ satisfy $\left.\mathbf{u}^{h}\right|_{\Gamma}=\mathbf{u}_{0}^{h}$ and $\left.T^{h}\right|_{\Gamma_{0}}=g^{h}$, respectively.

\subsection{Boundary Control Problems and Reduced-Order Control Problems.} Let $U$ be the control space defined by

$$
U=\left\{g=\sum_{i=1}^{p} g_{i} \chi_{i}, g_{i} \in R\right\}
$$

where $\chi_{i}$ is the $i$-th basis function of $U$ and is the trace of a $H^{1}(\Omega)$ function onto $\Gamma_{0}$. We consider the minimization of the form

$$
\min \|\nabla \times \mathbf{u}\|_{\Omega}^{2} \quad \text { subject to }(5.3) \text { or }(5.7)
$$

where the vorticity $\nabla \times \mathbf{u}$ is defined by $\nabla \times \mathbf{u}=\frac{\partial u_{2}}{\partial x_{1}}-\frac{\partial u_{2}}{\partial x_{2}}$ and the cost functional defines the total friction forces in $\Omega$.

We define the reduced basis element by the finite element approximation $(5.3)^{h}$ and $(5.7)^{h}$, respectively for each control problem. For example, the Lagrange reduced basis element $(\mathbf{u}, p, T)$ given $g^{j} \in U$ for problem (5.8) subject to (5.7) can be constructed by a solution $\left(\mathbf{u}^{h}, p^{h}, T^{h}\right)$ to $(5.7)^{h}$ given $g^{j} \in U$. For the case of the boundary control problem, the reduced basis space $X_{R} \subset \mathbf{X}^{h} \times X^{h}$ should consist of the basis element $\Phi_{0}^{h}$ that corresponds to the reference control $\bar{g} \in U$, the element $\Phi_{j}^{h}$ that corresponds to the $j$-th control in the direction of $\chi_{j}$, and the test functions $\Psi^{h} \in X_{R} \cap\left(\mathbf{X}_{0}^{h} \times X_{1}^{h}\right)$. Since $\mathbf{u}^{h}$ satisfies the pseudo-divergence condition $\left(\nabla \cdot \mathbf{u}^{h}, q^{h}\right)=0$ for all $q^{h} \in S^{h}$ we have the reduced-order control problem;

$$
\begin{aligned}
& \min \quad\left\|\nabla \times \mathbf{u}^{h}\right\|_{\Omega}^{2} \quad \text { subject to } \\
& \begin{aligned}
\frac{1}{R e}\left(\nabla \mathbf{u}^{h},\right. & \left.\nabla \mathbf{v}^{h}\right)+b_{0}\left(\mathbf{u}^{h}, \mathbf{u}^{h}, \mathbf{v}^{h}\right)+\left(\beta T^{h} \mathbf{g}, \mathbf{v}^{h}\right) \\
& +b_{1}\left(\mathbf{u}^{h}, T^{h}, \psi^{h}\right)+\kappa\left(\nabla T^{h}, \nabla \psi^{h}\right)=0
\end{aligned}
\end{aligned}
$$

for all $\Psi^{h}=\left(\mathbf{v}^{h}, \psi^{h}\right) \in X_{R} \cap\left(\mathbf{X}_{0}^{h} \times X_{1}^{h}\right)$. Here, the element in $X_{R}$ is represented by

$$
\left(u^{h}, T^{h}\right)=\Phi_{0}^{h}+\sum \alpha_{i} \Psi_{i}^{h}+\sum_{j=1}^{p} \frac{\left(g_{j}-\bar{g}_{j}\right)}{\delta_{j}} \Phi_{i}^{h}
$$


where $\Phi_{0}^{h}=\left(u^{h}, T^{h}\right)_{0}$ is a solution to $(5.7)^{h}$ corresponding to the reference control, $\Phi_{j}^{h}=$ $\left(u^{h}, T^{h}\right)_{j}-\left(u^{h}, T^{h}\right)_{0}$ for $1 \leq j \leq p$, with $\left(u^{h}, T^{h}\right)_{j}$ being a solution to $(5.7)^{h}$ corresponding to $\bar{g}+\delta_{j} \chi_{j}$ and $\left\{\Psi_{i}^{h}\right\}$ are a basis of the test function space $X_{R} \cap\left(\mathbf{X}_{0}^{h} \times X_{1}^{h}\right)$.

Similarly, for problem (5.8) subject to (5.3) we have the reduced-order control problem;

$$
\begin{aligned}
& \min \quad\left\|\nabla \times \mathbf{u}^{h}\right\|_{\Omega}^{2} \quad \text { subject to } \\
& \begin{aligned}
\frac{1}{R e}\left(\nabla \mathbf{u}^{h},\right. & \left.\nabla \mathbf{v}^{h}\right)+b_{0}\left(\mathbf{u}^{h}, \mathbf{u}^{h}, \mathbf{v}^{h}\right)+N\left(\nabla \phi^{h}-\mathbf{u}^{h} \times \mathbf{B}, \mathbf{v}^{h} \times \mathbf{B}\right) \\
& +\left(\nabla \phi^{h}-\mathbf{u}^{h} \times \mathbf{B}, \nabla \psi^{h}\right)=0,
\end{aligned}
\end{aligned}
$$

for all $\Psi^{h}=\left(\mathbf{v}^{h}, \psi^{h}\right) \in X_{R} \cap\left(\mathbf{X}_{0}^{h} \times X_{1}^{h}\right)$.

6. Computational Results. In this section we will give computational result by implementing the computational procedure for a specific control problem in channel flows using the two proposed control mechanisms. We select the backward facing step channel for our study, a schematic of this geometry is given in Figure 1. It has been observed in a number of computational and experimental study that a recirculation appears near the corner region for large Reynolds number. Our aim is to remove the recirculation by means of boundary control.

6.1. Boundary Temperature Control. The aim is to shape the flow to a desired configuration which in our study means to remove the recirculation by means of controlling the temperature along the bottom part of the boundary.

We assume that the inflow and outflow are parabolic, i.e. we take the inflow to be $\mathbf{u}_{i}=8(y-0.5)(1-y)$ and the outflow to be $\mathbf{u}=\mathbf{u}_{o}=y(1-y)$. We take the Reynolds number to be 200 and $\frac{G r}{R e^{2}}$ to be 1 . For the temperature we used the following boundary conditions:

$$
\begin{aligned}
& \Gamma_{s_{1}} \text { and } \Gamma_{\text {out }}: \quad \frac{\partial T}{\partial n}=0 \\
& \Gamma_{s_{2}} \text { and } \Gamma_{\text {bottom }}: T=g \\
& \Gamma_{\text {in }} \text { and } \Gamma_{\text {top }}: T=1 .
\end{aligned}
$$

Figure 2 qualitatively demonstrate the situation for high Reynolds number. Here our objective is to remove the recirculation that occurs in the corner region $\Omega^{*}$. Therefore we minimize vorticity in the corner region $\Omega^{*}$. This leads us to a constrained minimization problem of the type (5.8) and we use the reduced basis computational method described in $\S 5.4$.

Basis elements are computed with $g=1,0.875,0.75,0.625,0.5,0.3775,0.25$ and denoted by $\left(\mathbf{u}_{i}, T_{i}\right), i=1, \ldots, 7$. The test functions $\left\{\Psi_{1}, \ldots ., \Psi_{5}\right\}$ are chosen so that they have zero boundary conditions. The trial function $\Phi_{1}$ corresponds to the control force such that $\Phi_{1}=0$ everywhere on the boundary except on the bottom.

Then we set

$$
(\mathbf{u}, T)=\Phi_{0}+\frac{(g-\bar{g})}{\delta} \Phi_{1}+\sum_{i=1}^{5} \alpha_{i} \Psi_{i},
$$


where $\bar{g}=1, \delta=-0.75$ and

$$
\begin{gathered}
\Phi_{0}=\left(\mathbf{u}_{1}, T_{1}\right), \quad \Phi_{1}=\left(\mathbf{u}_{7}, T_{7}\right)-\left(\mathbf{u}_{1}, T_{1}\right), \\
\Psi_{1}=\left(\mathbf{u}_{7}, T_{7}\right)-2\left(\mathbf{u}_{6}, T_{6}\right)+\left(\mathbf{u}_{5}, T_{5}\right), \quad \Psi_{2}=\left(\mathbf{u}_{6}, T_{6}\right)-2\left(\mathbf{u}_{5}, T_{5}\right)+\left(\mathbf{u}_{4}, T_{4}\right), \\
\Psi_{3}=\left(\mathbf{u}_{5}, T_{5}\right)-2\left(\mathbf{u}_{4}, T_{4}\right)+\left(\mathbf{u}_{3}, T_{3}\right), \quad \Psi_{4}=\left(\mathbf{u}_{4}, T_{4}\right)-2\left(\mathbf{u}_{3}, T_{3}\right)+\left(\mathbf{u}_{2}, T_{2}\right), \\
\Psi_{5}=\left(\mathbf{u}_{3}, T_{3}\right)-2\left(\mathbf{u}_{2}, T_{2}\right)+\left(\mathbf{u}_{1}, T_{1}\right) .
\end{gathered}
$$

We finally employ Newtons method to the necessary optimality condition (4.4) to compute the control. We obtained the boundary temperature control $T=0.516$ in 7 Newton iterations. The computed control was then used in the full system to simulate the flow. The resulting flow shown in Figure 3 shows significant reduction in the size of the recirculation region.

6.2. Electromagnetic Control. In this problem, control is effected through boundary electric potential on the top boundary and on the bottom boundary of the backward facing step channel. A magnetic field $\mathbf{B}=(0,0,1)$ is applied into the fluid. The boundary conditions for the velocity are the same as in the preceding control problem except for the electric potential whose boundary conditions are as follows:

$$
\begin{aligned}
& \Gamma_{s_{2}}, \Gamma_{\text {in }} \text { and } \Gamma_{\text {out }}: \quad \frac{\partial \phi}{\partial n}=0 \\
& \Gamma_{\text {top }}: \phi=g_{1} \\
& \Gamma_{\text {bottom }}: \quad \phi=g_{2} \\
& \Gamma_{s_{2}}: \phi=1 .
\end{aligned}
$$

We take the Reynolds number to be $R e=200$ and the interaction parameter to be $N=1$. The basis elements were computed with

$$
\left(g_{1}, g_{2}\right)=(1,1),(1,0.5),(1,0),(0.5,1),(0.5,0.5),(0.5,0),(0,1),(0,0.5)
$$

and the corresponding elements are denoted by $\left(\mathbf{u}_{i}, \phi_{i}\right), i=1, \ldots, 8$. The test functions $\left\{\Psi_{1}, \ldots, \Psi_{5}\right\}$ are chosen so that they have zero boundary conditions. The trial functions $\Phi_{1}$ and $\Phi_{2}$ corresponds to the control force such that $\Phi_{1}=0$ everywhere on the boundary except on the top and $\Phi_{2}=0$ everywhere on the boundary except on the bottom. Then we set

$$
(\mathbf{u}, \phi)=\Phi_{0}+\frac{\left(g_{1}-\bar{g}_{1}\right)}{\delta_{1}} \Phi_{1}+\frac{\left(g_{2}-\bar{g}_{2}\right)}{\delta_{2}} \Phi_{2}+\sum_{i=1}^{5} \alpha_{i} \Psi_{i},
$$

where $\bar{g}_{1}=\bar{g}_{2}=1, \delta_{1}=\delta_{2}=-0.5$ and

$$
\Phi_{0}=\left(\mathbf{u}_{1}, \phi_{1}\right), \quad \Phi_{1}=\left(\mathbf{u}_{3}, \phi_{3}\right), \quad \Phi_{2}=\left(\mathbf{u}_{7}, \phi_{7}\right),
$$




$$
\begin{gathered}
\Psi_{1}=\left(\mathbf{u}_{1}, \phi_{1}\right)-2\left(\mathbf{u}_{2}, \phi_{2}\right)-2\left(\mathbf{u}_{4}, \phi_{4}\right), \quad \Psi_{2}=\left(\mathbf{u}_{1}, \phi_{1}\right)-\left(\mathbf{u}_{3}, \phi_{3}\right)-\left(\mathbf{u}_{7}, \phi_{7}\right), \\
\Psi_{3}=\left(\mathbf{u}_{1}, \phi_{1}\right)-\left(\mathbf{u}_{5}, \phi_{5}\right)-\left(\mathbf{u}_{2}, \phi_{2}\right)-\left(\mathbf{u}_{4}, \phi_{4}\right), \quad \Psi_{4}=\left(\mathbf{u}_{1}, \phi_{1}\right)-\left(\mathbf{u}_{8}, \phi_{8}\right)-\left(\mathbf{u}_{2}, \phi_{2}\right), \\
\Psi_{5}=\left(\mathbf{u}_{1}, \phi_{1}\right)-\left(\mathbf{u}_{6}, \phi_{6}\right)-\left(\mathbf{u}_{4}, \phi_{4}\right) .
\end{gathered}
$$

We employed the Newtons method to the necessary optimality condition (4.4) and obtained the boundary controls $\phi_{\text {top }}=1.0423$ and $\phi_{\text {top }}=1.7735$ respectively, in 5 Newton iterations. The computed control was then used in the full system to simulate the flow. The resulting flow shown in Figure 4 shows significant reduction in the size of the recirculation region.

In conclusion, we have demonstrated the feasibility of using reduced basis method in both one parameter and two parameter control setting. Our numerical results seem to indicate that the reduced basis method can be successfully used in flow control problems with significant reduction in computational cost compare to the results presented in [9] for the same problems where computations were performed by directly applying finite element methods to the optimal control problems.

\section{REFERENCES}

[1] B.O. Almroth, P. Stern and F.A. Brogan, Automatic Choice of Global Shape Functions in Structural Analysis, AIAA Journal, 16, (1978), 525-528.

[2] M. Desai And K. ITo, Optimal Control of Navier-Stokes Equations, SIAM J. Control \& Optim., 32 (1994), 1428-1446.

[3] V. Girault and P. Raviart, Finite Element Methods for Navier-Stokes Equations (Springer-Verlag, New York, 1986).

[4] K. Ito and S.S. Ravindran, A Reduced Order Method for Simulation and Control of Fluid Flows, J. Computational Physics (submitted) .

[5] K. Ito and S.S. Ravindran, Optimal Control of Thermally Convected Flows, SIAM J. Scientific Computing (submitted).

[6] A.K. Noor, Recent Advances in Reduction Methods for Nonlinear Problems, Computers \& Structures, 13 (1981), 31-44.

[7] A.K. Noor, C.M. Anderson and J.M. Peters, Reduced Basis Technique for Collapse Analysis of Shells, AIAA Journal, 19 (1981), 393-397.

[8] J.S. Peterson, The Reduced Basis Method for Incompressible Viscous Flow Calculations, SIAM J.Sci. Stat. Comput., 10 (1989), 777-786.

[9] S.S. Ravindran, Computations of Optimal Control for Fluid Flows, To appear in Optimal Control of Viscous Flows, S. S. Sritharan (Ed.), Frontiers in Applied Mathematics, SIAM, 1996.

[10] J.S. Walker, Large Interaction Parameter Magnetohydrodynamics and Applications in Fusion Reactor Technology; in Fluid Mechanics in Energy Conversion, J. Buchmaster, Ed., SIAM, Philadelphia, 1980 . 


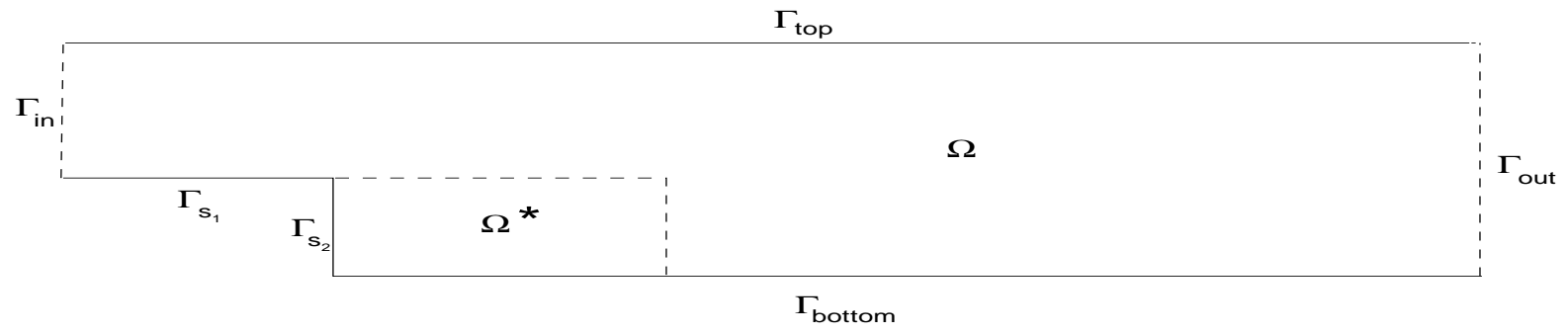

FIG. 1. Schematic of backward-facing-step channel.

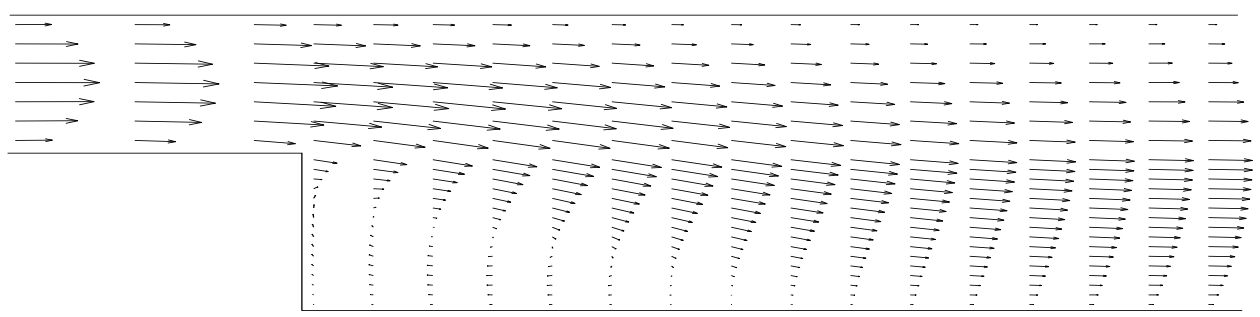

FIG. 2. Uncontrolled velocity field at $\operatorname{Re}=200$ 


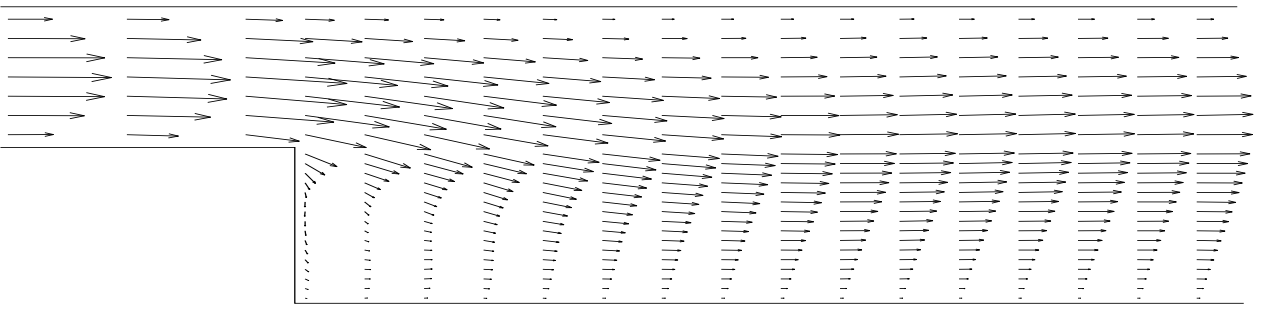

FIG. 3. Controlled velocity field at $\mathrm{Re}=200$

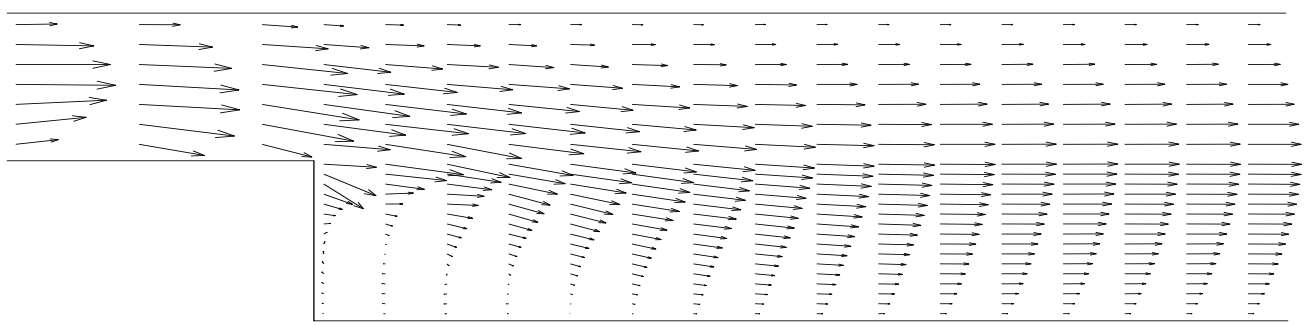

FIG. 4. Controlled velocity field at $R e=200$ 